\title{
Mauer, Heike, und Johanna Leinius (Hrsg.) (2021): Intersektionalität und Postkolonialität. Kritische feministische Perspektiven auf Politik und Macht
}

\section{Opladen/Berlin/Toronto: Verlag Barbara Budrich. 301 Seiten. $52 €$}

\section{Ina Kerner 10}

Angenommen: 20. Dezember 2021 / Online publiziert: 11. Januar 2022

(C) Der/die Autor(en) 2022

Intersektionalität und Postkolonialität bzw. - etwas geläufiger - Postkolonialismus sind zwei überlappende Forschungsrichtungen mit unterschiedlicher Genealogie, die bezogen auf die Forschung zu Politik und Geschlecht Ähnliches beanspruchen: kritische Analysen zu leisten von Rassismus und von Kolonialität, das heißt von kolonialen Hinterlassenschaften und Aktualisierungen, und zwar in ihren jeweiligen Verflechtungen mit Geschlechterverhältnissen. Der Begriff Intersektionalität wurde im Kontext des Schwarzen Feminismus geprägt und bezeichnet das komplexe Zusammenspiel vor allem rassistischer und sexistischer, potenziell aber auch weiterer hierarchisierender, stratifizierender und segregierender Strukturen, Institutionen, Diskurse, Denkmuster und Handlungsweisen. Der Begriff Postkolonialismus ist kritisch auf den europäischen Kolonialismus mit seiner Vielfalt rassistischer und gewaltförmiger Praktiken bezogen, die von Enteignung und Willkürherrschaft bis hin zu Sklaverei und Genozid reichen.

Der 2021 erschienene, aus einer Tagung im Herbst 2017 entwickelte und unbedingt lesenswerte Sammelband greift beide Traditionen auf und führt sie in drei Rubriken zusammen: „Begriffsarbeit“, „Staat und Institutionen“ und „Soziale Bewegungen“. Dabei ist auffällig, dass sich die meisten der Beiträge entweder mit intersektionalen Perspektiven oder mit postkolonialen Ansätzen befassen, die sie dann jeweils auf politikwissenschaftliche Forschungsfelder beziehen, auf die sie bislang selten bezogen werden; ein Beispiel ist die Biopolitik, in diesem Fall die Forschung an menschlichem Erbgut und Medikamententests, die Helene Gerhards in ihrem Kapitel intersektional diskutiert. Allerdings bleibt der Band bei einer bloßen Zusammenstellung intersektionaler und postkolonialer Herangehensweisen nicht stehen. In

Ina Kerner $(\triangle)$

Seminar Politische Wissenschaft, Institut für Kulturwissenschaft, Universität Koblenz-Landau,

Campus Koblenz, Koblenz, Deutschland

E-Mail: kerner@uni-koblenz.de 
den Beiträgen der Herausgeberinnen Heike Mauer und Johanna Leinius werden sie vergleichend verhandelt, in einem Gespräch zwischen Nikita Dhawan und Birgit Sauer über analytischen Mehrwert, politologische Rezeption und Fallstricke beider Ansätze werden sie in einen Dialog gebracht. Ferner gibt es in dem Band Texte, die noch einen Schritt weitergehen und beide Stränge auf interessante Weise verknüpfen. Das ist etwa der Aufsatz von Antje Daniel über queerfeministische Problematisierungen männlicher Dominanz in der südafrikanischen Studierendenbewegung der letzten Jahre, also der im Zusammenhang von \#RhodesMustFall angestoßenen Bewegung für eine Dekolonisierung der Hochschulen; oder der abolitionistische Text von Sonja John über indigene Frauen und staatliche Gefängnispraktiken in Kanada, in dem sie das „Gefängnis als fortdauernde[n] Siedlungskolonialismus“ (S. 148) problematisiert und diese Problematisierung mit einer intersektionalen Analyse der Inhaftierung und ihrer Effekte zusammenlaufen lässt.

Und auch der Umstand, dass in diversen Buchbeiträgen die intersektionale Kerntrias Race/Class/Gender explizit erweitert wird - um Indigenität, um queere Perspektiven, aber auch um Religion - ist richtungsweisend und zeigt die große Bandbreite der Themen und Perspektiven, die im Zeichen von Intersektionalität und Postkolonialismus inzwischen in der deutschsprachigen Politikwissenschaft zusammenkommen.

Hält man sich diese Bandbreite vor Augen, mag es auf den ersten Blick erstaunen, dass sich die beiden Herausgeberinnen in ihren drei eigenen Beiträgen der Aufgabe eines Vergleichs bzw. einer Verhältnisbestimmung der beiden Forschungsstränge widmen. Denn andere Aufsätze des Bandes zeigen ja gerade, dass eine Abgrenzung zwischen Intersektionalität und Postkolonialismus zunehmend schwierig wird, weil beide Perspektiven nicht nur in der Forschung, sondern auch in der politischen Praxis zunehmend zusammengedacht werden. Dennoch sind die Beiträge von Leinius und Mauer äußerst instruktiv. Denn sie demonstrieren nicht nur die Heterogenität und die große Aktualität beider Forschungsperspektiven - letzteres exemplifiziert anhand von \#BlackLivesMatter und den komplexen politischen Managementversuchen und Effekten der Coronapandemie. Ferner umreißen die Beiträge, auch für Leser*innen mit wenig Vorkenntnissen, zentrale Stationen der Rezeptionsgeschichte beider Perspektiven und zeichnen nach, wie hier jeweils mit einem alten Problem feministischer Theorie und Praxis verfahren wird, nämlich dem Umgang mit Gleichheit und Differenz in einer von Ungleichheit und diversen Bias geprägten Welt, der affirmative Bezugnahmen auf bestehende Kategorisierungen zuweilen nötig macht, auch wenn das Fernziel die Auflösung der dahinter stehenden Kategorisierungssysteme sein mag. In ihrem Schlusskapitel diskutieren Leinius und Mauer ferner aktuelle Debatten der intersektionalen und der postkolonialen Forschung. Dabei geht es um die letztlich auch hochschulpolitische Frage, wer solche Forschung betreibt, betreiben sollte und $\mathrm{zu}$ betreiben eingestellt wird; um die Frage, wie im deutschen Sprachraum mit der Kategorie „Rasse“ umzugehen ist; und um die bislang erst am Rande diskutierte Rolle des Antisemitismus in intersektionalen und postkolonialen Analysen.

Zusammenhänge von Macht, Politik und Religion stehen erfreulicherweise in drei Aufsätzen explizit im Zentrum des Bandes - auf sehr unterschiedliche Weise, auch wenn es in jedem der drei Beiträge in besonderem Maße um den Islam geht. Rich- 
tungsweisend ist hierbei Zubair Ahmads an Arbeiten u.a. von Talal Asad orientierter Hinweis darauf, dass die Religionen, wie wir sie in der Regel diskutieren, ein stark am Christentum modelliertes Konstrukt sind, das selbst eine machtkritische Genealogie gut vertragen kann, und zwar von der europäischen Kolonialgeschichte bis in die globalisierte Gegenwart. Floris Biskamp arbeitet sich an dem Problem ab, dass die Kritik des Sexismus minorisierter Religionen als Rassismus wirken oder gar explizit mit rassistischer Absicht erfolgen kann, und entwickelt auf Grundlage von Arbeiten der postkolonialen Theoretikerin Gayatri Spivak einen Kriterienkatalog für die Unterscheidung zwischen legitimer Kritik und rassifizierenden Formen des Othering. Bei diesen Kriterien geht es darum, ob homogenisiert, viktimisiert und entpolitisiert wird oder nicht; wie es um den Wahrheitsgehalt einer Darstellung bestellt ist; und ob die Sprecher*innenposition transparent gemacht wird. Und so plausibel Biskamps Kriterien erscheinen mögen, regen sie doch zu weiterem Nachdenken an: Nämlich darüber, wie weit man mit einem aus der politiktheoretischen Vogelperspektive entworfener Kriterienkatalog im nachvollziehbarerweise hochgradig affektiv aufgeladenen Feld von Sexismus, Rassismus und Rassismusvorwürfen überhaupt kommen kann; und ob eine weitere Reflexionsschleife zur Sprecher*innenposition - eine Reflexionsschleife, bei der die Position der Kriterien entwerfenden Theoretiker*in im Zentrum steht - eine andere Form der kritischen Auseinandersetzung nahelegen könnte, etwa eine stärker am konkreten Fall orientierte und weniger abstrakt evaluierende Form. Christopher Fritzsche schließlich befasst sich mit Bezugnahmen auf den Islam durch antifeministische Akteur*innen, genauer gesagt auf strategische Indienstnahmen und strategische Bündnisse im weiten Feld gegenwärtiger AntiGender-Politiken, das rechte Parteien und fundamentalistische Zweige der unterschiedlichsten Religionsgemeinschaften vereint. Der Islam erscheint insbesondere rechtskatholischen und US-evangelikalen Aktivist*innen gegen die Ausweitung der Ehe auf gleichgeschlechtliche Paare als Bündnispartner - obwohl rechte Politiken in Europa oftmals explizit durch eine antimuslimische Haltung geprägt sind und dabei nicht zuletzt geschlechter- und familienpolitische Fragen in den Mittelpunkt stellen, wie etwa das Heiratsalter und Polygamie.

Neben den Beiträgen von Fritzsche und Daniel befasst sich ein weiteres Kapitel des Bandes auf innovative Weise mit sozialen Bewegungen. Christine Löw lenkt den Blick auf subalterne indische Frauen und deren politische Analysen und Kämpfe an der Schnittstelle von neoliberaler Globalisierung, Klimaschutz und Ernährungssicherheit. Zu den interessanten Ergebnissen ihrer ausführlichen Diskussion gehört die Betonung eines Zusammenhangs zwischen Kolonialismus und den Effekten des Klimawandels, einschließlich dessen politischem Steuerungsversuch durch Instrumente des Waldschutzes im globalen Süden und des globalen Emissionshandels beide haben indigene Frauen auf besondere Weise prekarisiert.

Ein dritter Beitrag über „Staat und Institutionen“ ist neben den Aufsätzen von John und Gerhards eine Abhandlung über intersektionale Ungleichheiten im ökonomisierten deutschen Wohlfahrtsstaat der Koautorinnen Monika Götsch und Katrin Menke. Inhaltlich geht es hier um zwei Fälle, nämlich zum einen um das Erleben von Wahlfreiheit bezogen auf die Verbindung reproduktiver und beruflicher Tätigkeiten von erwerbstätigen Eltern im Krankenhaussektor und zum anderen um Erfahrungen von Transpersonen im Erwerbskontext. Das kaum überraschende, dennoch wichtige 
Ergebnis lautet, dass diese Wahrnehmungen und Erfahrungen nicht einheitlich sind, sondern sich je nach sozialer Positionierung, etwa der Klasse oder der Herkunft, unterscheiden.

Der bislang noch unerwähnte vierte Beitrag in der Buchrubrik „Begriffsarbeit“, der ansonsten neben einem Kapitel der Herausgeberinnen die Texte von Ahmad und Biskamp umfasst, stammt von Laura Mohr und eruiert die politische und theoretische Produktivität einer Verbindung intersektionaler und queerer Ansätze. Eine solche Produktivität lokalisiert Mohr vor allem im Zusammenspiel des gesellschaftskritischen Potenzials beider Perspektiven, die auf unterschiedliche Weise auf Differenzierungs- und Normierungseffekte in kapitalistischen Gesellschaften bezogen sind. „Queere Intersektionalität“ zielt nach Mohr auf gesellschaftliche Transformationsprozesse und die hierfür nötigen Formen politischer Solidarität ab, die von einem Interesse an Normen- und Normalitätskritik ebenso geleitet sein müssen wie von einem positiven und dennoch nichtessenzialisierenden Bezug auf bewegungsinterne Differenzen.

Dass der Band „Intersektionalität und Postkolonialität“ als heterogen charakterisiert werden kann, als Zusammenschau ganz unterschiedlicher Perspektiven, ist evident; dass er politisch relevante, politikwissenschaftlich bislang dennoch oft vernachlässigte Themen und Perspektiven in die Diskussion bringt, ist hoffentlich ebenfalls klar. Wie hingegen der Umstand zu bewerten ist, dass fast alle Kapitel des Buches von Personen verfasst wurden, die prekär oder befristet beschäftigt sind, ist eine implizite Frage des Bandes an die Politikwissenschaft als verfasster Disziplin. Man kann diese Frage als intersektionale oder auch als postkoloniale Frage verstehen. Eine Beantwortung steht aus.

Funding Open Access funding enabled and organized by Projekt DEAL.

Open Access Dieser Artikel wird unter der Creative Commons Namensnennung 4.0 International Lizenz veröffentlicht, welche die Nutzung, Vervielfältigung, Bearbeitung, Verbreitung und Wiedergabe in jeglichem Medium und Format erlaubt, sofern Sie den/die ursprünglichen Autor(en) und die Quelle ordnungsgemäß nennen, einen Link zur Creative Commons Lizenz beifügen und angeben, ob Änderungen vorgenommen wurden.

Die in diesem Artikel enthaltenen Bilder und sonstiges Drittmaterial unterliegen ebenfalls der genannten Creative Commons Lizenz, sofern sich aus der Abbildungslegende nichts anderes ergibt. Sofern das betreffende Material nicht unter der genannten Creative Commons Lizenz steht und die betreffende Handlung nicht nach gesetzlichen Vorschriften erlaubt ist, ist für die oben aufgeführten Weiterverwendungen des Materials die Einwilligung des jeweiligen Rechteinhabers einzuholen.

Weitere Details zur Lizenz entnehmen Sie bitte der Lizenzinformation auf http://creativecommons.org/ licenses/by/4.0/deed.de. 